\title{
Study and Application of Advanced PLC Control Strategy
}

\author{
Baoxiang Yang \\ Xi'an Aeronautical Polytechnic Institute, Xi'an China 710089, China
}

Keywords: advanced PLC control strategy; PLC study; PLC application.

\begin{abstract}
Along with unceasing economic development and the progress of science and technology, PLC, as a common control system, is used more and more widely. Additively, as one of the commonest mainstream control systems, PLC's superior anti-jamming capability and multifunctional development way will get better and better. The process of industrialization is accelerating today and the controlled objects are becoming more and more complex. In this context, a variety of technologies should be better integrated into the PLC development. By giving specific analysis to the study and application of advanced PLC control strategy, the author hopes to provide some reference opinions and suggestions for others.
\end{abstract}

\section{Introduction}

PLC is the abbreviation of programmable logic controller. At present, the application of PLC is only limited to some control fields like data collection, the order control and PID feedback. Moreover, although the PLC algorithm is considerably stability, its control process is often very complex. Therefore, the study of PLC control strategy is of significance. The author specifically analyzes the advanced control functions, study and application of PLC in this paper, based on relevant information access and data analysis. By fully using the experience of developed countries for reference, the author gives analysis to successful experience, aiming to make the entire PLC program system can be better extended and ultimately achieve better results 1 .

\section{Concept of PLC}

Programmable logic controller, short for PLC, is a digital operating system specifically designed to be used in industrial environment. It is a programmable memory widely used at this stage. The main instructions that it can execute include logic operation, sequential control, timing and instructions of other aspects. These instructions are finally output through the digital simulation system to control the production process of all kinds of mechanical equipment better.

\section{Development of PLC}

The microprocessor appeared as early as in 1970s, and then programming logic controller was introduced soon. Thus, the programmable logic controller enables operations, data transfers and other functions to be included into the function of programming logic control more quickly, ultimately realizing the assembly of industrial control devices which truly have computer characteristics. The combination of microcomputer technology and relay jointly forms the logic controller, and programmable logic controller is eventually named PLC with the development of personal computers.

Until the late 1970s, programmable logic devices finally achieved a phased development and the technology of programming controller has made a qualitative leap. On this basis, the reliable anti-interference simulator with higher computing speed and smaller size was developed, and the performance of PID is also becoming better.

Subsequently, until the 1970s and 1980s, the growth rate of PLC reached $30 \%-40 \%$ per year. In this period, the PLC's analog processing, ability of digital computing and man-machine docking as well as the capability of major network were greatly improved. This shows that the entire PLC field has entered a higher stage. 
Today, the entire PLC is better adapted to the development of modern industry while more man-machine interaction interfaces have come into beings, making various PLCs get better development in industrial control equipment ${ }^{2}$.

\section{Basic structure of PLC}

The PLC is actually a kind of logic controller which can carry out programming. It is specially used in industrial control and its hardware and software structure is as the same as the structure of micro-computer. The basic structure mainly includes the following aspects:

\subsection{Power supply}

The process of converting AC directly into DC required by PLC is called the power supply. At present, the power supply mode adopted by most kinds of PLCs is switching regulated power supply.

\subsection{Central processing unit}

The center of the entire PLC is called the central processing unit (CPU), which directly determines the performance of the entire PLC. The PLC is mainly composed of the controller, arithmetic unit, register and many other parts. These electronic components are not concentrated on the same chip. By connecting the address bus and the control bus with the memory as well as electrical equipment in the inlet and outlet, the CPU can better control the whole running process of users. This logic calculation process is very similar to that of mathematics.

\subsection{Memory}

The tool with a powerful memory of semiconductor circuit is called a memory. Storage system procedures, user programs and logical variables are its main functions, and its main basic role is to achieve program functions of the entire PLC better. Most of the production code for PLCs is written by their manufacturers and eventually solidified into ROM memory. Most users are not able to access it.

\subsection{Input unit}

The entire input interface connected to the PLC device is called the input unit. It is a bridge that the signal flows into the PLC and its main role is to receive the master components and detect various signals from components. DC input, AC input and AC/DC input are three most dominant modes of the input unit.

\subsection{Output unit}

The connection element between the PLC and controlled devices is referred to as the output unit. It is the component which transmits signal output from the PLC directly to a controlled device. This component mainly converts weak electric signals sent by CPU into the level signals. It is an actuating element of controlled devices. Relay output, transistor output and crystal gate output is the main part of the entire drive equipment.

\section{Functional characteristics of PLC}

\subsection{High reliability}

PLC mostly employs the operation pattern of single-chip microcomputer. Seen from the overall structure, it has high integration and the function of circuit protection function. Its automatic diagnosis function can judge the situation of the entire internal system, further enhancing reliability of the whole system.

\subsection{Being easy to be programmed}

PLC programming mostly adopts the ladder diagram and command language of relay control, and the quantity is much less than those which micro-machine uses. Except for the middle-end and high-end PLCs popular now, small PLCs generally contain only about 16 programming procedures. In addition, as the whole ladder graphics is fairly simple, PLC is quite easy to be mastered and applied. Even the personnel without very professional knowledge of programming can carry out programming ${ }^{3}$. 


\subsection{Flexible programming configuration}

The overall components of the PLC adopt a modular structure. Through simple combination, users can change functions of the entire system while the whole control of system functions can also become easier. Therefore, PLC is suitable for the control of any system.

\subsection{Complete module for input and output functions}

DC/AC switches, digital value, analog quantity, voltage and current in different fields as well as other types of on-site signals can be directly connected to the relevant devices in the industrial field via different templates, and are eventually connected to the motherboard via the bus.

\subsection{Convenient installation}

The installation of the entire PLC does not need to be carried out in a professional room and professional shielding measures are not required in the installation process. In the process of use, it can work properly when the testing device is correctly connected with corresponding implementation interface.

\subsection{High operation speed}

The entire control process of PLC is carried out by programs. So it not only has high operation speed, but also has unparalleled reliability. These are the manifestations that PLC is more advanced than the relay logic control.

\section{Application of advanced PLC control strategy}

\subsection{Fuzzy database and its rules}

If the fuzzy database can combine with the rule base better, the fuzzy controller will have more objectives. All the language variables and functions exist in the database. The scale transition factor and space of overall input and output need to be divided accordingly. Moreover, the rules of the entire PLC usually need to be described by language variables before they form a set of fuzzy rules. Experts' knowledge, rules in the rule base as well as other experience and knowledge are all those which have been reflected in specific areas. For the fuzzy control system, knowledge base is indispensable. In addition, for the entire field of fuzzy control system, fuzzy reasoning machine is its core. It has dual concept of reasoning, namely simulating human beings and making concept fuzzier.

In accordance with the entire fuzzy rules, the fuzzy reasoning machine has a very wide application scope. It uses fuzzy rules in the knowledge base to carry out fuzzy reasoning, and thereby get final induction according to relations and reasoning methods of relevant fuzzy logic. To have such a dual function, the functions of knowledge base and reasoning system in the entire fuzzy system must have close relationship with each other. In the controlling process, the overall fuzzy controller can be divided into the single variable controller and multivariable controller, according to the overall output and input functions. And finally the angle of the entire output mode can be divided into position-based output and incremental output. The whole control system can have better effects from the beginning to the end only when the proper form of fuzzy controller can be selected according to the practical use.

\subsection{Communication module of intelligent network}

The entire programmable controller is developed on the basis of sequential logic control module. Structurally, it is a device which is specifically designed for manipulation and digital operations in industrial environments, and thus the PLC can also be called microcontrollers for industrial control. It mainly has two characteristics. For one thing, its programming is very convenient, so that it is used quite widely in the industrial environment with complex operational sequence; for the other thing, the whole structure is made up of the development achievements of large-scale integrated circuit, microcomputer and address book, eventually forming series of products with various advantages and in different size. And then, it can be applied into different fields of control system together with the relay controller.

In recent years, PLC intelligent modules are constantly being developed and the entire Internet communication capability is also strengthened constantly. In order to constantly meet requirements of various control systems, more and more modules with different functions are being constantly 
developed. A lot of modules popular now belong to a part of the high speed counting module, such as the high speed counting module, temperature control module, remote control module, communication module, human-computer interface module, and so on. These modules are usually equipped with CPU and intelligent memory, which makes the function of PLC be further extended. Finally, due to PLC's flexible and convenient functions, its application is also expanding to larger scope. This is the future development trend of PLC technology.

\subsection{Structure of fuzzy neural network}

The essence of the so-called fuzzy control is a control technology simulating human mind. It is a kind of control rules by which the control experience and knowledge can be truly transformed into language variables. Making use of fuzzy information and various logical reasoning patterns, a variety of physical parameters are used to compare the physical meanings. Although this approach can get clear meanings, it does not have the ability of mutual learning and adjustment. The artificial neural network does not have good ability of knowledge expression, but it eventually can realize the function simulation of the entire biological neural network. The whole simulating process has a very strong ability of self-study and adaption, and can also deal with errors better ${ }^{4}$.

It can be seen from the analysis above that the fuzzy control function and neural network function often have a strong performance of mutual complementarities. These two functions can eventually achieve good complementarities and ultimately realize the integration and application of the overall neural network. This is a process of learning from each other, which can help the two to play their advantages better and ultimately build a control system more complete and intelligent. These two intelligent control systems, the fuzzy network and neural network, can make the overall learning functions and adaptability become more powerful through a better combination. And only when the fuzzy control rules can be better optimized can good control effect be achieved finally.

\section{Application of PLC}

\subsection{Control of switches}

PLC has a very powerful application of PLC switches and the number of points it covers in the whole control process can be from a dozen to dozens and even to several hundred. If the entire control ability of PLC is strong enough, it can even control tens of thousands of points. Furthermore, the entire process of PLC operation can be networked, and its points are not subject to the overall control of the switch. PLC can be widely used in a lot of industries, such as the metallurgy, machinery, chemical industry, light industry, and so on. The control targets of PLC cannot be compared with other machines. Therefore, PLC also can be used in control of switches more easily.

\subsection{Control of analog quantity}

As a large industrial electronic control device, PLC's biggest shortage is that it cannot control various quantities. To this end, PLC manufacturers have made a lot of efforts for development in this aspect. Actually, medium-sized machines can control the analog quantity and so can small machines.

In addition, PLC can be well integrated with A/D and D/A combination in the process of analog control, implements the control of entire PID fuzzy algorithm and ultimately makes the whole control volume becomes better. The biggest advantage of analog control with a PLC is that it can control switches while conducting analog quantity control. This is the unique control advantage of PLC, but this is not very convenient to achieve.

\subsection{Control of digital quantity}

PLC is a computer-based control technology. With its increasingly perfect development, it is very convenient to achieve overall digital control by it. The entire PLC can receive the operation of the data pulse, so that the frequency can become very high. And it can be received through a variety of types of lines in the process of operation. Many PLCs even have the function of simplest pulse output. Finally, coupled with the PLC's data processing functions and computing power, it can eventually fully realize the various control principles of NC. 


\section{Conclusion}

Overall, with the development of the times, PLC will become more and more advanced. It is also believed that the advanced control strategy of PLC will become better and better.

\section{References}

[1] Tang Peili, Zhao Qiang. PLC Control Technology in Frequency Conversion Water Supply System [J]. China New Technologies and Products, 2013 (12): 123.

[2] Tian Hai, Cui Guimei, Wang Xiaohong et al. Configuration Strategy and Application of PLC Control Network in Siemens [J]. Electrical Drive, 2010 (1): 76-80.

[3] Lu Yan, Luo Qinghua, Wei Kexin. The Fuzzy PID Controller Achieved by PLC and Its Application in the System of Blast Furnace Burden Distribution [J]. Journal of Tianjin University of Technology, 2008 (2): 141.

[4] Jiang Jianfang. PLC Engineering Application Technology Tutorial of Siemens S7-200 [M]. Beijing: China Machine Press, 2009. 